\title{
Estrategia para el fortalecimiento y seguimiento de las competencias genéricas en el contexto universitario*
}

\author{
Carolina Salamanca Leguizamón \\ Sandra Milena Neira Camacho ${ }^{2}$ \\ Eulalia Medina Díaz ${ }^{3}$
}

Recibido: 09-03-2020

Aceptado: 14-05-2020

\section{Resumen}

El presente artículo da a conocer resultados de la investigación "Fortalecimiento del desarrollo de competencias genéricas en estudiantes universitarios a partir de un modelo de evaluación externa", desarrollada desde el enfoque cualitativo, con diseño de investigación-acción educativa con docentes del programa de Licenciatura de Educación Infantil de la Fundación Universitaria de San Gil (Unisangil). Como resultado se diseñó una estrategia educativa para el fortalecimiento de las competencias genéricas en estudiantes de programas académicos de una institución de educación superior colombiana, en especial para el diseño y aplicación de actividades de aula desde la actualización de documentos que soportan el currículo y una guía para orientar

\footnotetext{
* Artículo resultado de proyecto de investigación realizado en Unisangil.

1. Magíster en Educación. Docente universitaria de Unisangil (San Gil, Colombia), grupos de investigación ICES y Tarepe, líneas de investigación: pedagogía y prácticas pedagógicas, educación y pedagogía.

Correo: csalamanca1@unisangil.edu.co

ORCID: https://orcid.org/0000-0002-4344-0538
}

2. Magíster en Educación especialidad en Educación Superior. Docente universitaria de Unisangil (San Gil, Colombia), grupo de investigación Tarepe, líneas de investigación: pedagogía y producción de textos escritos y formación para la ciudadanía.

Correo: sneira@unisangil.edu.co

ORCID: https://orcid.org/0000-0001-6703-7519

3. Magíster en Educación con especialidad en Educación Superior. Decana de la Facultad de Ciencias de la Educación y de la Salud y docente investigadora universitaria de Unisangil (San Gil, Colombia), grupo de investigación ICES, línea de investigación: pedagogía y prácticas pedagógicas.

Correo: emedina@unisangil.edu.co

ORCID: https://orcid.org/0000-0003-0200-5297 
el proceso. Se concluyó que hacer uso tanto de las competencias genéricas propuestas por el Icfes como de las competencias asumidas por la IES, en el diseño de sílabos y planes de trabajo, resulta útil para la ejecución y seguimiento de actividades de aula y de los aprendizajes.

Palabras clave: competencias genéricas, currículo, estrategia didáctica, sílabos.

\title{
Strategy for strengthening and monitoring generic competences in the university context
}

\begin{abstract}
This article reports the results of the research "Strengthening the development of generic competences in university students from an external evaluation model", developed from a qualitative approach, with design of educational research-action with teachers of the Bachelor's degree program Early Childhood Education of the San Gil University Foundation (Unisangil). As a result, an educational strategy was designed to strengthen generic competencies in students of academic programs of a Colombian higher education institution, especially for the design and application of classroom activities from the updating of documents that support the curriculum and a guide. to guide the process. It was concluded that making use of both the generic competences proposed by the Icfes and the competences assumed by the IES, in the design of syllables and work plans, is useful for the execution and monitoring of classroom activities and learning.
\end{abstract}

Keywords: generic competences, curriculum, didactic strategy, syllables. 


\section{Introducción}

Desde hace más de una década, en Colombia las instituciones académicas de orden superior han adquirido el compromiso de desarrollar competencias genéricas (2009) en sus estudiantes, razón por la cual desde el 2004 la institución de educación superior (IES) en la que se realizó esta investigación (Unisangil) inició un proceso de aproximación hacia la formación y evaluación por competencias específicas. La institución, a través de jornadas pedagógicas, generó espacios de reflexión que permitieran poner en contexto el modelo pedagógico institucional para responder a las necesidades y las demandas actuales.

Dada la forma única como sucede el desarrollo y adquisición de las competencias, una de las formas estandarizadas de evaluar los estadios de desarrollo de estas ha sido a través de las pruebas de Estado, creadas y administradas por el Instituto Colombiano para la Evaluación de la Educación (Icfes). Aprovechando esta estructura, en el año 2017, docentes investigadoras de la IES realizaron un estudio con el fin de identificar el valor agregado (Icfes, 2020) obtenido del resultado de las pruebas de Estado como mecanismo para evaluar la calidad de un programa para la misma IES.

El análisis de los resultados obtenidos a través de la recolección de la información acerca de la percepción de las competencias que creen usar en el desarrollo de las asignaturas, suministrada tanto por estudiantes, como por docentes de la IES, puso en evidencia que, si bien la institución cuenta con los direccionamientos para la inclusión de las competencias genéricas en la planeación de sus clases, falta generar estrategias de aula para desarrollarlas, lo cual fue confirmado por los docentes durante las jornadas de capacitación realizadas a nivel institucional(Gómez \& Ostos, 2018). Por esta razón se hizo necesario generar una estrategia que orientara el

\footnotetext{
4. Los modelos de valor agregado son modelos estadísticos que buscan cuantificar cuánto aportan las instituciones educativas a sus estudiantes en términos de aprendizaje, independientemente de las condiciones de entrada de los alumnos, teniendo en cuenta factores institucionales e individuales. Actualmente estos modelos son una herramienta convencional en la literatura internacional sobre medición de la calidad de la educación.
} 
fortalecimiento y seguimiento de las competencias genéricas que tenga en cuenta los aportes del marco de referencia suministrado por el Icfes para la evaluación de la calidad de la educación superior.

El proyecto de investigación aquí presentado se desarrolló entre los años 2018 y 2019, y se planteó como objetivo principal diseñar una estrategia educativa para el fortalecimiento de las competencias genéricas como indicadores de aprendizaje de los estudiantes de programas académicos de esta IES, a partir del marco de referencia de evaluación del Icfes y en coherencia con los lineamientos curriculares institucionales. El presente artículo da a conocer los referentes teóricos y conceptuales, los aspectos metodológicos, y los resultados y hallazgos más significativos de la propuesta.

\section{Acercamientos teóricos y conceptuales}

Los referentes que orientaron la propuesta de investigación se asocian con la concepción de currículo, el desarrollo de las competencias y especialmente el desarrollo de competencias genéricas, así como con la concepción de los indicadores de desempeño y la reflexión pedagógica como ejercicio permanente en el quehacer del maestro.

De acuerdo con el análisis acerca de la concepción de currículo realizado por Caicedo y Calderón (2016), hay tres categorías: los centrados en la organización del saber (planes), en la organización de la acción (experiencias) y en la organización de la complementariedad del saber y la acción (planes y acciones). En la primera categoría encontramos concepciones como las de Dewey (1973), para quien el currículo guía y da estructura al proceso de enseñanzaaprendizaje; mientras que para Stenhouse (1984) se trata de un intento de comunicar los principios y características de un propósito educativo que posibilite su eficaz traslado a la práctica, y para Gimeno (1994) es el conjunto de temas que se abordan desde las disciplinas aproximando conocimientos y aportes sobre la educación. 
En relación con las definiciones que se centran en las experiencias y la organización de la acción, se encuentra el concepto de Sarramora (1987, citado por Achury, 2012), quien define el currículo como el conjunto de actividades realizadas en los centros educativos con el fin de conseguir el desarrollo de los jóvenes, un objetivo general de la educación en la mayoría de las sociedades occidentales.

Por su parte, Gimeno (1994) propone cinco ámbitos para analizar el currículo: por su función social, como proyecto o plan educativo, como la expresión formal y material de ese proyecto, como un campo práctico y como una actividad discursiva. Esta mirada amplia de currículo permite ir más allá de entenderlo en cuanto programa o plan de estudio, pues integra las demás posibilidades de aprendizaje, orientadas a los conocimientos conceptuales y procedimentales, pero también al desarrollo de capacidades y a fortalecer las actitudes.

\section{Zabalza (1987) expresa que}

es un conjunto de los supuestos de partida, de las metas que se desea lograr y los pasos que se dan para alcanzarlas; el conjunto de conocimientos, habilidades, actitudes, etc., que se considera importante trabajar en la escuela años tras años. Y por supuesto la razón de cada una de esas opciones. (p. 37)

Pansza (1990) reconoce en el currículo una cadena estructurada de conocimientos y experiencias de aprendizaje que se articulan con una clara intención de generar aprendizajes que permitan pensar y actuar frente a los problemas cotidianos de la vida social y laboral: "constituye el qué y el cómo que se enseña, presenta dos aspectos diferenciados y al mismo tiempo interconectados: la construcción y la acción que se articulan a través de la evaluación" (p. 42).

Grundy (1998, citado por Álvarez, 2010), considera el currículo como: 
parte integrante de la cultura de la sociedad. Para comprender el significado de cualquier conjunto de prácticas curriculares, han de considerarse tanto en cuanto a elementos que surgen a partir de un conjunto de circunstancias históricas, como en calidad de reflejo de un determinado medio ambiente de aprendizaje. (p. 74)

Por su parte, en la categoría de currículo centrado en la organización de la complementariedad del saber y de la acción, cabe el concepto establecido por el Ministerio de Educación Nacional de Colombia, que en la Ley General de Educación (1994) define el currículo como el

conjunto de criterios, planes de estudio, programas, metodologías y procesos que contribuyen a la formación integral y a la construcción de la identidad cultural nacional, regional y local, incluyendo también los recursos humanos, académicos y físicos para poner en práctica las políticas y llevar a cabo el proyecto educativo institucional. (Ley 115 de 1994, art. 76)

Santiváñez (2013) señala que, pese a la extensa gama de definiciones que hay para la palabra currículo, todas coinciden en un conjunto de elementos básicos, a saber, "educandos, representantes sociales, objetivos, contenidos y experiencias de aprendizaje" (p. 34). Además de todo lo que hay en común, este mismo autor expresa que el currículo lo constituyen las experiencias de aprendizaje que viven los estudiantes, dentro y fuera del ámbito educativo, bajo la orientación del maestro, atendiendo al propósito de alcanzar fines y objetivos de la educación. Las experiencias de aprendizaje pueden obedecer a una programación o también pueden surgir desde la cotidianidad educativa, atendiendo a la permanente interrelación entre institución educativa y comunidad. 


\section{Desarrollo de competencias en el contexto educativo}

Si bien el concepto de competencia surge en el ámbito laboral (Mertens, 2000), este fue ganando espacio en el contexto educativo para responder a los retos impuestos a la educación por el sistema socioeconómico. Al hablar de competencias, los planes curriculares deben especificar un para qué, las actividades de aprendizaje y enseñanza, así como la evaluación de estas. "La formación por competencias se da desde el desarrollo y fortalecimiento de habilidades de pensamiento como clave para formar personas éticas, emprendedoras y competentes" (Hernández y Poncio, 2018, p. 149).

Dentro del marco de este proyecto, se asumió la definición de competencia como:

Procesos complejos de desempeño con idoneidad en determinados contextos, integrando diferentes saberes (saber ser, saber hacer, saber conocer y saber convivir), para realizar actividades $\mathrm{y} / \mathrm{o}$ resolver problemas con sentido de reto, motivación, flexibilidad, creatividad, comprensión y emprendimiento, dentro de una perspectiva de procesamiento metacognitivo, mejoramiento continuo y compromiso ético, con la meta de contribuir al desarrollo personal, la construcción y afianzamiento del tejido social, la búsqueda continua del desarrollo económicoempresarial sostenible, y el cuidado y protección del ambiente y de las especies vivas. (Tobón, 2008, p. 5)

Vinculadas a la práctica de elaborar diseños curriculares profesionales, se han propuesto algunas taxonomías de competencia, a fin de establecer los procedimientos progresivos de aprendizaje, ejemplo de ello es el proyecto Tuning (2013), desde el cual se clasifican las competencias como genéricas y específicas.

En Colombia, la formulación de las competencias genéricas para la educación superior constituye una tarea que el Ministerio de 
Educación Nacional (MEN)emprendió en 2008, como una de sus estrategias para establecer referentes comunes que garanticen la calidad de la formación en el país. Así que, en una de sus publicaciones, el MEN asegura que formular dichas competencias

busca responder a las necesidades del mundo globalizado, en el que las distancias se han acortado con los medios de comunicación y de transporte, las fronteras económicas y laborales tienden a difuminarse y la educación busca cada vez con más fuerza la internacionalización de los saberes, las profesiones, las titulaciones y los mercados de trabajo. Sin descuidar, por supuesto, la pertinencia frente el contexto local, para el cual estas competencias también constituyen una fuente de desarrollo, de modo que su implementación contribuirá al mejoramiento de la calidad de la formación y a su proyección internacional. (2009, p. 1)

En respuesta a este propósito, en el año 2008 el Ministerio organizó las competencias genéricas en cuatro grandes grupos, así: comunicación en lengua materna y en otra lengua internacional; pensamiento matemático; ciudadanía, y ciencia, tecnología y manejo de la información (MEN, 2009).

De acuerdo con el Proyecto Educativo Institucional de la Fundación Universitaria de San Gil (2018), que basó su definición de competencia en lo expresado en el Proyecto Educativo Tuning (2007) y los lineamientos del Ministerio de Educación Nacional (2006), las competencias son capacidades que todo ser humano necesita para resolver de manera autónoma las situaciones de la vida, saber hacer en la vida y para la vida, saber ser, saber emprender y saber vivir en comunidad, y trabajar en equipo. En coherencia con los avances que a la fecha ha hecho el Ministerio de Educación Nacional en el tema de competencias, esta IES las clasifica en: competencias específicas y competencias básicas. Las específicas están asociadas a la profesión y, por tanto, preparan al estudiante para cumplir con éxito las responsabilidades propias del ejercicio profesional, teniendo en cuenta que estas, tal como lo plantea Gimeno (2008), 
constituyen conglomerados de aspectos, no solo cognitivos, sino también actitudinales, de motivación y de valores. Son conjunciones de condiciones integradas de los sujetos que permanecen como cualidades o rasgos latentes de las personas [...]. No son capacidades fijas ni definitivas, sino algo cambiante, que evoluciona, no cerrado. (p. 46)

Por su parte, las competencias básicas son comunes entre programas de formación inscritos en un campo disciplinar. En el caso de las licenciaturas, y las ingenierías son aquellas que comparte con los licenciados, sin importar su énfasis o área del conocimiento en la que es titulado. Para su construcción, se sugiere tener en cuenta los mismos criterios mencionados anteriormente. $Y$ las competencias genéricas, entendidas como las que, independientemente de la profesión, le permiten al futuro profesional de la IES donde se desarrolló la investigación, responder a los retos del mundo actual en el ámbito personal, social, académico y laboral. Son comunes a todos los programas de formación.

Vale la pena señalar que no todas las asignaturas podrán trabajar todas las competencias genéricas, por ello conviene que cada una se comprometa a abordar aquellas que pueda desarrollar dados el objeto de la disciplina, la metodología que utiliza y la función que la disciplina tiene en el plan de estudios. Además, debe estar mencionada en el sílabo y en la planeación del docente la competencia genérica a desarrollar, y se requiere mostrar los criterios de evaluación de estas, así como la estrategia de seguimiento constante para garantizar su desarrollo desde los procesos de aprendizaje (Gómez, 2019).

Lo anterior les profiere a estas competencias un abordaje gradual y constante durante el proceso formativo del profesional y asegura en buena medida mejores desempeños en todas las asignaturas y hasta en las pruebas de Estado.

Respecto a los indicadores de desempeño, estos se reconocen como acciones complejas que permiten desarrollar y demostrar las 
competencias. Se distinguen de las conductas porque no son respuestas definidas a situaciones preestablecidas, sino que exigen la construcción o aplicación a partir de los conocimientos para abordar situaciones demandantes que desafíen el pensamiento rígido y esquemático con el que pueden llegar al aula los estudiantes (Santiváñez, 2013). Es importante diferenciar los indicadores de desempeño de las actividades de aprendizaje, aunque en ocasiones puedan coincidir. Por ejemplo, hacer una revisión bibliográfica para identificar las principales tendencias en marketing internacional puede ser una actividad, mientras que el desempeño puede estar relacionado con analizar las principales tendencias en marketing internacional aplicables al marketing local.

\section{Reflexión pedagógica}

Desde principios del siglo $\mathrm{XX}$, John Dewey hizo contribuciones significativas acerca del profesor como un profesional reflexivo. Dewey reconoció en el profesor un profesional con la capacidad de desempeñar un papel activo en la construcción de conocimiento. Para abordar el concepto de reflexión desde este autor, es preciso ahondar en su propuesta, es decir, pensar la experiencia sobre dos componentes: el ensayar, componente activo, y el padecer, o componente pasivo.

De manera que la práctica que tiene una intención, que plantea unos objetivos de transformación, corresponde con el componente activo, y establece un diálogo con el segundo componente, el pasivo (Dewey, 1989). Así pues, la reflexión se origina en la práctica, ya que nace de una situación que es vivida.

Dewey (1989) define la reflexión como "el examen activo, persistente y cuidadoso de toda creencia o supuesta forma de conocimiento a la luz de los fundamentos que la sostienen y las conclusiones a las que tiende" (p. 25). La postura de Dewey en torno a la reflexión fue retomada por Donald Schön, quien hizo cuestionamientos importantes a los modelos de formación de los profe- 
sionales en Europa y Norteamérica en los años 70. El autor situó su análisis en la racionalidad técnica imperante en los centros superiores de formación, que generaba una formación relacionada con la apropiación de saberes y técnicas producidas por comunidades académicas y científicas, lo cuales luego eran aplicados en campos y situaciones particulares de desempeño profesional.

En palabras de Schön (1992):

La racionalidad técnica defiende la idea de que los profesionales de la práctica solucionan problemas instrumentales mediante la selección de los medios técnicos más idóneos para determinados propósitos. Los profesionales de la práctica que son rigurosos resuelven problemas instrumentales bien estructurados mediante la aplicación de la teoría y la técnica que se derivan del conocimiento sistemático, preferiblemente científico. (p. 17)

Lo que este autor demuestra es que el modelo descrito no responde a las realidades de los profesionales, ya que, en el terreno de la práctica, ocurren hechos que no son resueltos solo con el saber que ha sido acumulado. Además, la autonomía se ve amenazada, porque desde este enfoque se genera una dependencia con el saber establecido. Se desconocen pues las bondades de formar profesionales autónomos capaces de reflexionar en la acción y sobre la acción. Para Schön la reflexión "está en el interior del desarrollo permanente, según la propia experiencia, las competencias y los conocimientos profesionales de cada uno" (1992, p. 12). De manera que estos estudios llevaron a Schön a proponer que la práctica se genera y se estudia como ruta en la formación profesional. Desde allí se privilegia el diálogo, más que la instrucción, y el análisis de condiciones que hay en el ejercicio de la profesión.

Para el caso del profesional docente, resulta muy valiosa la concepción de esta epistemología de la práctica, ya que no se orienta solo a aprender el hacer, sino también a un hacer reflexivo. Retomando las palabras de Pérez, Roa, Villegas y Vargas (2013), este hacer reflexivo "implica un distanciamiento de la acción y una posi- 
ción permanente de alerta académica. Este enfoque posibilita pensar la sistematización como una particular manera de investigar para transformar la práctica y para transformarse a sí mismo" (p. 7).

Lo anterior reafirma que la sola práctica en los contextos de aula no es garantía de un aprendizaje crítico de lo vivido, tampoco lo es la sola exploración de alternativas, ni las intervenciones "adecuadas" de aula que se hagan; en muchas ocasiones no son más que reproducciones tradicionales de modelos docentes tradicionales.

\section{Metodología}

La investigación se enmarcó en el paradigma crítico-social con un enfoque cualitativo. El diseño es de investigación-acción educativa, en el cual

la búsqueda de conocimiento se entiende como un proceso colectivo, cuyos resultados y la utilización de los mismos son obtenidos por los colectivos, quienes determinan el proceso de conocimiento a la vez que experimenta, en el mismo, un progreso en la maduración colectiva que permite asumir el control de los problemas que los afectan. (Bautista, 2011, p. 94)

Una de las técnicas usada para esta investigación fue el análisis documental, que se constituye como una herramienta apropiada para recopilar y analizar la documentación suministrada por las fuentes secundarias, a saber, los sílabos y planes de trabajo, que conformaron la información objeto de estudio. Adicionalmente se hizo observación y registro de actividades de clase, como también encuestas, con el fin de recolectar información para la valoración de la puesta en práctica de las estrategias pedagógicas diseñadas por los docentes, a través de cuestionarios estructurados.

La investigación se desarrolló en tres fases: la primera, de actualización de formatos y construcción de un documento guía de tra- 
bajo, la segunda fase de formación docente y una tercera fase de implementación de actividades y seguimiento de resultados (figura 1). En cada una se desarrollaron actividades orientadas a construir una estrategia institucional que permita, por medio de documentos guía del proceso, realizar el diseño, aplicación y seguimiento de actividades de clase para docentes de la IES implicada en esta investigación, así como orientar el trabajo con docentes para el diseño de actividades de clase y propuesta de metodologías para el registro y sistematización de las actividades académicas implementadas con miras al fortalecimiento de competencias genéricas de los estudiantes de un programa académico piloto de la misma IES.

Figura 1. Fases de desarrollo de la investigación.

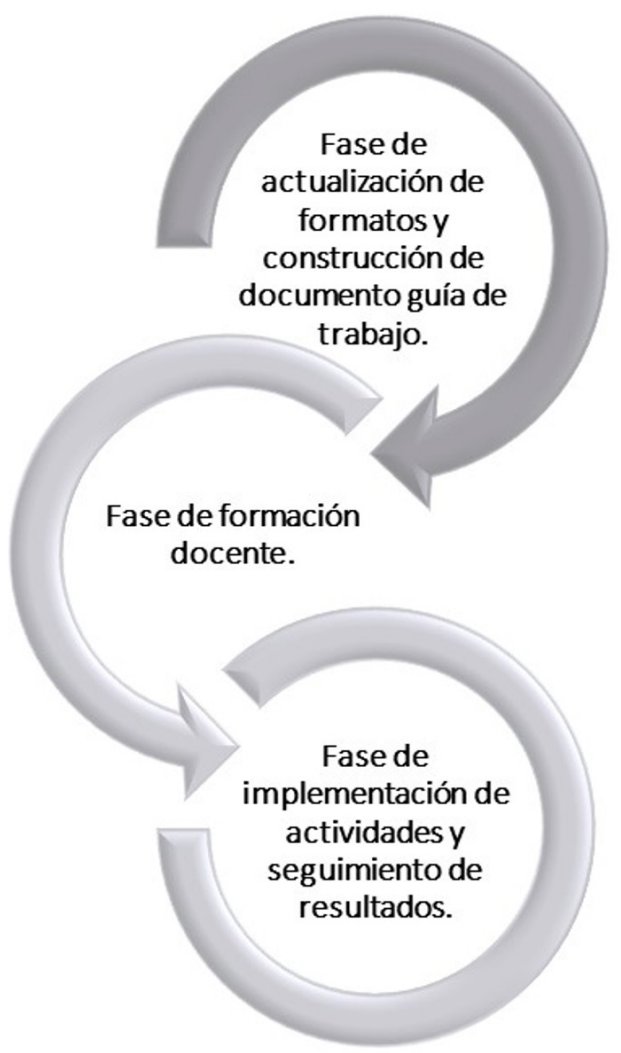

Fuente: Elaboración propia. 


\section{Resultados}

\section{Documento guía del proceso de diseño, aplicación y seguimiento de actividades}

Para la elaboración del documento guía del proceso de diseño, aplicación y seguimiento de actividades fue necesario, en primer lugar, realizar un cruce entre las siguientes competencias genéricas propuestas por el Ministerio de Educación Nacional (MEN) que, hasta el momento en que inició este proyecto, tenía como referencia la IES: lectura crítica, competencias ciudadanas, comunicación escrita, razonamiento cuantitativo e inglés, con las competencias genéricas propuestas por el Icfes. Para las demás competencias genéricas propuestas por el MEN se realizó la clasificación de acuerdo con la diferenciación que hace la IES entre contenidos conceptuales, procedimentales y actitudinales (ver tabla 1), derivada del enfoque de competencias, en el que la solución de problemas complejos es posible con la combinación de distintos elementos, entre ellos procesos de pensamiento, teorías, estrategias, métodos y actitudes. Los contenidos conceptuales son los relacionados con el saber; en ellos se abordan conceptos, teorías, fenómenos, leyes, enunciados, modelos, hechos y principios (para esta investigación son aquellos que el estudiante aprende por medio de las competencias genéricas y específicas). Los procedimentales están referidos al saber hacer, comprenden la ejecución de habilidades, estrategias, técnicas o métodos y pasos para realizar acciones. $Y$ los actitudinales están asociados al saber ser; en estos se abordan valores, normas, actitudes, principios éticos, entre otros.

Tabla 1. Clasificación de las competencias genéricas como contenidos esenciales.

\begin{tabular}{|c|c|c|}
\hline Contenidos conceptuales & Contenidos procedimentales & Contenidos actitudinales \\
\hline Lectura crítica & $\begin{array}{c}\text { Razonamiento analítico y } \\
\text { sintético }\end{array}$ & Entendimiento interpersonal \\
\hline Competencias ciudadanas & Pensamiento creativo & Trabajo en equipo \\
\hline Comunicación escrita & Solución de problemas & \\
\hline
\end{tabular}




\begin{tabular}{|c|c|c|}
\hline Contenidos conceptuales & Contenidos procedimentales & Contenidos actitudinales \\
\hline Razonamiento cuantitativo & Manejo de la información & \\
\hline Inglés & $\begin{array}{c}\text { Uso de las tecnologías de la } \\
\text { información y la comunicación }\end{array}$ & \\
\hline
\end{tabular}

Fuente: elaboración propia.

Una vez organizadas las competencias de acuerdo con la propuesta de la IES y teniendo en cuenta que el modelo de evaluación por evidencias del Icfes consta de unos niveles de complejidad del 1 al 4 , siendo 1 el menor y 3 el esperado, en cuanto a los desempeños alcanzados en cada competencia se propuso encaminar las actividades pedagógicas de acuerdo con los niveles de aprendizaje para los programas académicos, así: niveles de aprendizaje 1 y 2 , en un nivel de competencia 1 ; niveles de aprendizaje 3 y 4 , en un nivel de competencia 2; niveles de aprendizaje 5 y 6 , en un nivel de competencia 3; y niveles de aprendizaje $7,8,9$ y 10, en un nivel de competencia 4.

A partir de estas consideraciones se realizó una reestructuración de los componentes que conforman tanto el sílabo, como el plan de trabajos; estos ajustes respecto al sílabo se evidenciaron en uno de sus apartados denominado "Orientaciones metodológicas", pues se agregó orientación para el proceso de evaluación del aprendizaje; asimismo fue ampliada la información presentada en las competencias y los indicadores de desempeño, al agregar para las competencias genéricas y específicas las afirmaciones, la evidencia y la descripción, y respecto a los contenidos esenciales se adicionó el nombre de la unidad didáctica y el contenido.

En consecuencia, el documento Plan de Trabajo también presentó modificaciones en relación con la presentación y articulación de las competencias de la asignatura, el tiempo de desarrollo, los contenidos esenciales, las actividades, y la evaluación acorde a los criterios e instrumentos de evaluación.

\footnotetext{
5. Documento que retoma la propuesta curricular del programa de formación para la asignatura (sílabo); a partir de los elementos descritos allí, el docente organiza las actividades presenciales y de trabajo independiente que propondrá durante el semestre para orientar el proceso de aprendizaje de los estudiantes. Este documento constituye la planeación del ejercicio docente.
} 
En la tabla 2 se evidencia el encabezado resultante de la modificación del plan de trabajo, en el cual fue posible relacionar la mayoría de los elementos que se encontraban separados en el formato existente con el fin de diligenciarlo evidenciando la coherencia entre ellos.

Tabla 2. Formato del Plan de Trabajo.

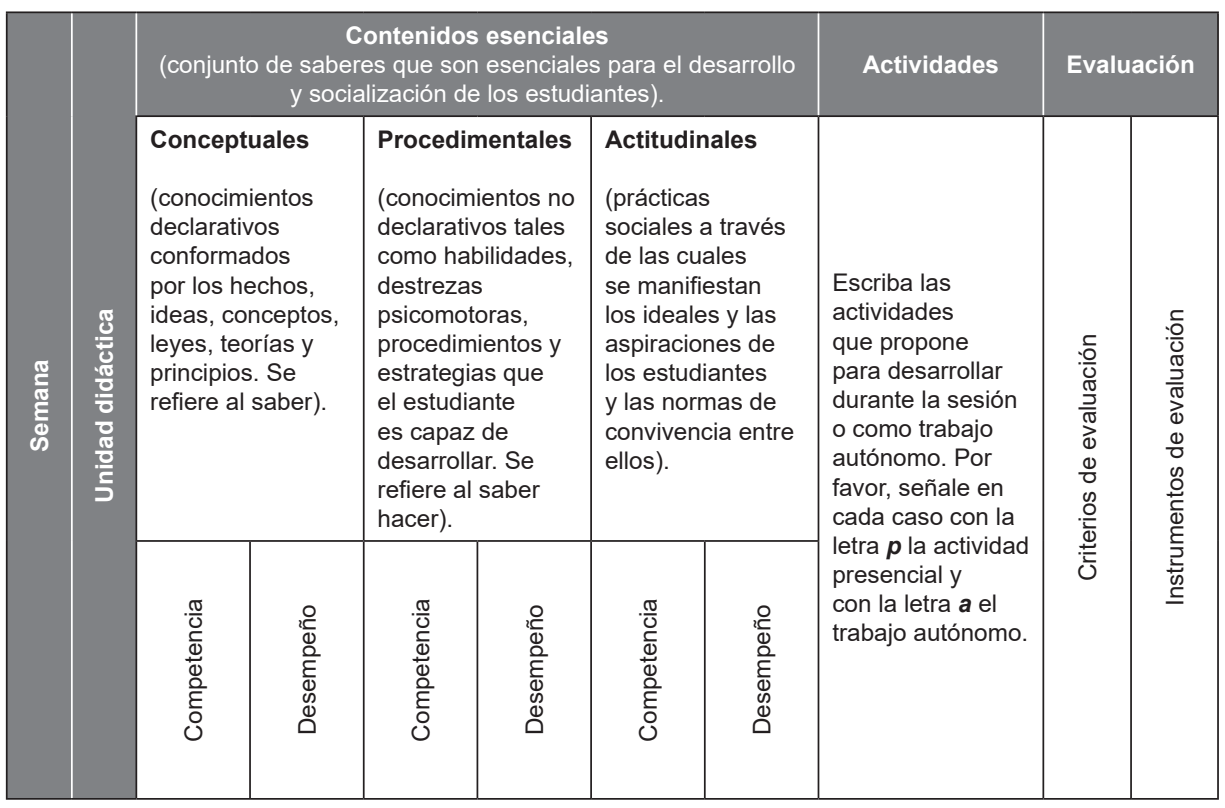

Fuente: elaboración propia.

A continuación, se realizó una modificación en los sílabos para todas las asignaturas del programa de Licenciatura en Educación Infantil (programa piloto), del primer al cuarto nivel de aprendizaje. Este ejercicio permitió la reflexión que dio lugar al documento guía de orientación para docentes, en el que se hizo una descripción de los elementos del plan de trabajo y el paso a paso para formular actividades de clase desde el ejercicio de las competencias genéricas, con una evaluación que evidenciara los avances que el estudiante presenta frente a cada una de ellas. La metodología descrita en el documento se sintetiza en la figura 2. 
Figura 2. Metodología para el diseño de actividades de clase centradas en competencias.

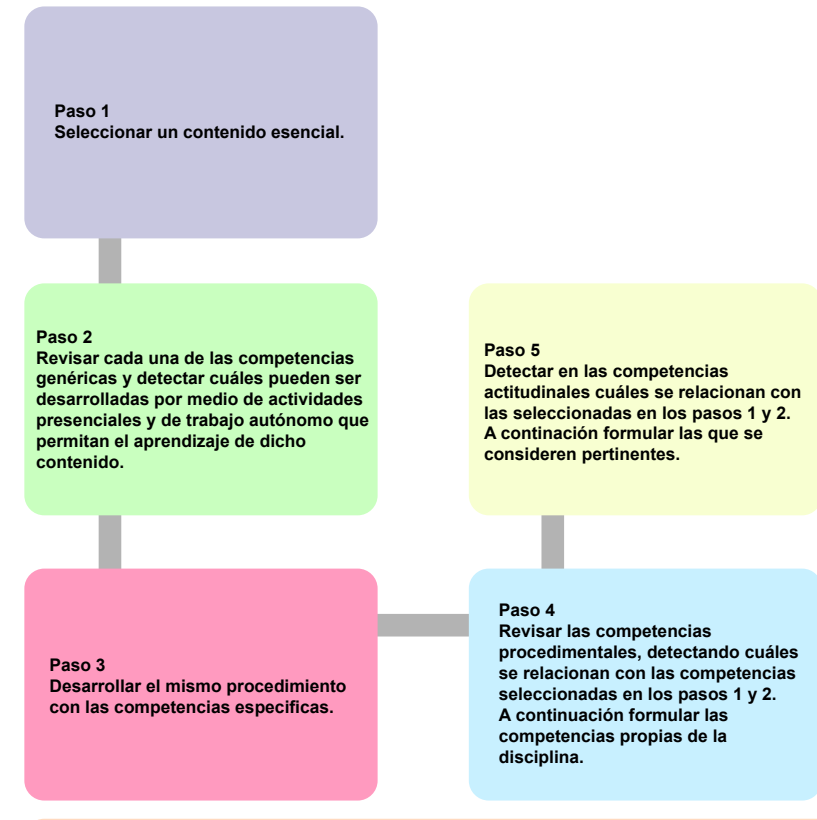

Considerar actividades evaluativas para definir los criterios y los instrumentos de evaluación. Tener presente la bibliografía requerida para el desarrollo de las competencias seleccionadas de acuerdo al plan de trabajo.

Fuente: elaboración propia.

\section{Trabajo con docentes para el diseño y aplicación de actividades de aula}

La propuesta de los sílabos y el plan de trabajo fue socializada con once docentes, dos de ellos con dedicación de tiempo completo al programa de Licenciatura en Educación Infantil y nueve catedráticos. Esto se llevó a cabo en un encuentro en el que además de presentar los avances del proyecto, cada docente exploró sus sílabos y actualizó la bibliografía. Adicionalmente, se acordó con cada uno el espacio que se tendría para planificar la sesión de clase (Ostos, 2017). 
A continuación, se llevaron a cabo encuentros personalizados con los docentes, con quienes fue posible planear nueve sesiones de clase en las asignaturas de Filosofía de la Educación, DesarroIlo Infantil, Historia del Pensamiento Pedagógico, Introducción a la Primera Infancia, Estimulación Temprana, Electiva Genérica 3: Habilidades Comunicativas, Sociología de la Educación, Expresión Oral y Didáctica General.

Al aplicar las sesiones diseñadas se solicitó a cada docente realizar una reflexión sobre la práctica; para ello cada uno diligenció un formato de reflexión de sesión de clase, y asimismo, en cada clase un estudiante diligenció un formato de observación de la jornada.

El seguimiento se centró básicamente en establecer en qué medida la aplicación de la sesión correspondió con el diseño; de igual manera, qué criterios e instrumentos de evaluación fueron utilizados, así como los aprendizajes obtenidos por parte de los estudiantes y los retos a los que se enfrentaron los docentes. Además, fue necesario realizar con algunos docentes una entrevista semiestructurada a fin de ampliar la información obtenida en los registros.

Para esta fase de trabajo fueron tenidas en cuenta dos unidades de análisis: la planeación de las actividades de aula y la ejecución de las actividades desde la perspectiva de los docentes y de los estudiantes; esta última se complementa con los resultados de una entrevista a los docentes.

Para realizar el análisis se establecieron cuatro categorías dentro de estas unidades: actividades, criterios de evaluación, instrumento, desempeño, con el fin de evaluar la estrategia diseñada. Los resultados de dicho análisis se sintetizan en la tabla 3. 
Tabla 3. Seguimiento del trabajo con maestros.

\begin{tabular}{|c|c|c|c|}
\hline \multirow{2}{*}{$\begin{array}{l}\text { Unidades } \\
\text { de análisis } \\
\text { Criterios }\end{array}$} & \multirow{2}{*}{$\begin{array}{l}\text { Planeación de las } \\
\text { actividades de aula }\end{array}$} & \multicolumn{2}{|c|}{ Ejecución de las actividades planeadas } \\
\hline & & $\begin{array}{l}\text { Entrevistas semiestructuradas } \\
\text { a docentes }\end{array}$ & $\begin{array}{l}\text { Observaciones de } \\
\text { clase realizadas por } \\
\text { estudiantes }\end{array}$ \\
\hline 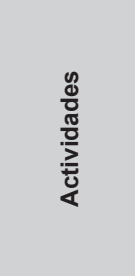 & $\begin{array}{l}\text { Los docentes, a partir } \\
\text { de una temática, } \\
\text { planearon actividades } \\
\text { que apuntan al } \\
\text { fortalecimiento de } \\
\text { las competencias } \\
\text { genéricas. }\end{array}$ & $\begin{array}{l}\text { Para la mayoría de los docentes } \\
\text { la ejecución de las actividades } \\
\text { se realizó conforme a lo } \\
\text { planeado; las variaciones a esta } \\
\text { generalidad corresponden a } \\
\text { adaptaciones que realizaron de } \\
\text { acuerdo con las necesidades } \\
\text { o situaciones particulares } \\
\text { presentadas. }\end{array}$ & $\begin{array}{l}\text { Para la mayoría de } \\
\text { los estudiantes la } \\
\text { reflexión se situó en } \\
\text { el procedimiento y las } \\
\text { actividades realizadas, } \\
\text { dando cuenta mediante } \\
\text { la descripción de lo } \\
\text { que los docentes } \\
\text { planearon. }\end{array}$ \\
\hline 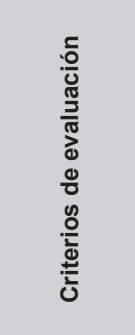 & $\begin{array}{l}\text { La planeación también } \\
\text { permitió reconocer la } \\
\text { coherencia entre las } \\
\text { unidades temáticas, } \\
\text { las competencias, } \\
\text { las actividades a } \\
\text { desarrollar, los criterios } \\
\text { de evaluación y los } \\
\text { instrumentos usados } \\
\text { para ello. }\end{array}$ & $\begin{array}{l}\text { No se obtuvieron resultados } \\
\text { homogéneos, es decir, algunos } \\
\text { docentes manifestaron en } \\
\text { su escrito coherencia entre } \\
\text { los criterios para evaluar el } \\
\text { aprendizaje de los estudiantes } \\
\text { con lo planeado, mientras que } \\
\text { otros no lo tuvieron en cuenta en } \\
\text { la reflexión de la actividad. }\end{array}$ & $\begin{array}{l}\text { Para la mayoría de los } \\
\text { estudiantes los criterios } \\
\text { de evaluación fueron } \\
\text { narrados como parte } \\
\text { de los aprendizajes } \\
\text { obtenidos. Los criterios } \\
\text { fueron relacionados } \\
\text { en algunas de las } \\
\text { actividades planeadas, } \\
\text { en otras no fueron } \\
\text { percibidos. }\end{array}$ \\
\hline 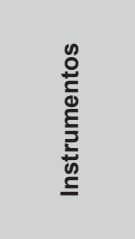 & & $\begin{array}{l}\text { La mayoría de los docentes } \\
\text { hicieron uso del instrumento que } \\
\text { se planeó; estos instrumentos } \\
\text { no fueron revisados, de manera } \\
\text { que no se puede afirmar que } \\
\text { correspondan con los criterios de } \\
\text { evaluación establecidos por los } \\
\text { docentes. }\end{array}$ & $\begin{array}{l}\text { En la mayoría de } \\
\text { las reflexiones de } \\
\text { los estudiantes no } \\
\text { se mencionaron } \\
\text { los instrumentos de } \\
\text { evaluación usados por } \\
\text { parte del docente. }\end{array}$ \\
\hline 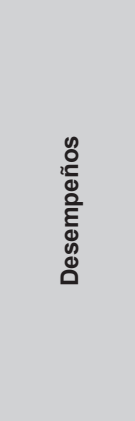 & & $\begin{array}{l}\text { Las competencias privilegiadas } \\
\text { en la descripción de su alcance } \\
\text { son aquellas que se encuentran } \\
\text { directamente relacionadas con } \\
\text { la actividad: escritura, lectura, } \\
\text { inglés. Con menor profundidad, } \\
\text { se encontraron el entendimiento } \\
\text { interpersonal y uso de las } \\
\text { tecnologías de la información y } \\
\text { la comunicación; casi ignoradas, } \\
\text { el manejo de la información, el } \\
\text { razonamiento analítico y sintético } \\
\text { y las competencias ciudadanas. }\end{array}$ & $\begin{array}{l}\text { Al consultarles a } \\
\text { los estudiantes } \\
\text { qué aprendieron, } \\
\text { la mayoría de las } \\
\text { respuestas se enfocó } \\
\text { en los contenidos o } \\
\text { temas que se trabajan } \\
\text { en la clase, y algunos } \\
\text { hacen referencia } \\
\text { a los desempeños } \\
\text { relacionados con } \\
\text { las competencias } \\
\text { de entendimiento } \\
\text { interpersonal. }\end{array}$ \\
\hline
\end{tabular}

Fuente: elaboración propia. 


\section{Estrategia educativa para el fortalecimiento de las competencias genéricas como indicadores de aprendizaje de los estudiantes}

La estrategia construida como producto del proceso descrito tiene por nombre "Al día con las competencias genéricas", en cuyo objetivo se planteó fortalecer las competencias genéricas como indicadores de aprendizaje de los estudiantes de programas académicos de la IES a partir del marco de referencia de evaluación del Icfes en coherencia con los lineamientos curriculares institucionales.

Respecto a la metodología se propuso, en primer lugar, consolidar un equipo de trabajo para cada programa académico, liderado por el comité curricular institucional, con el fin de desarrollar la estrategia. Este equipo de trabajo estaría integrado por un docente orientador, el director de programa e integrantes del programa de apoyo y seguimiento.

Entre las funciones del docente orientador están: asesorar al comité curricular en la actualización de los sílabos, así como a los docentes en el diseño de los planes de trabajo y el cargue de las experiencias a la plataforma de aprendizaje virtual de la institución educativa.

Por su parte, el director de programa convocará el encuentro entre los docentes del programa con el fin de reflexionar acerca de las experiencias pedagógicas y seleccionar la que recibirá reconocimiento en el homenaje al docente. De igual forma, organizará los simulacros de pruebas de competencias genéricas periódicamente.

El programa de apoyo y seguimiento académico registrará los resultados de las pruebas Saber 11; asimismo diseñará los simulacros de pruebas de competencias genéricas y registrará los resultados obtenidos, liderará las acciones de mejora para los estudiantes que registren dificultades en las pruebas, y finalmente registrará los resultados de las pruebas Saber Pro y generará el análisis de valor agregado. 
Las actividades propuestas a realizar en la estrategia se enuncian en la figura 3.

Figura 3. Actividades propuestas en la metodología de fortalecimiento de competencias genéricas.

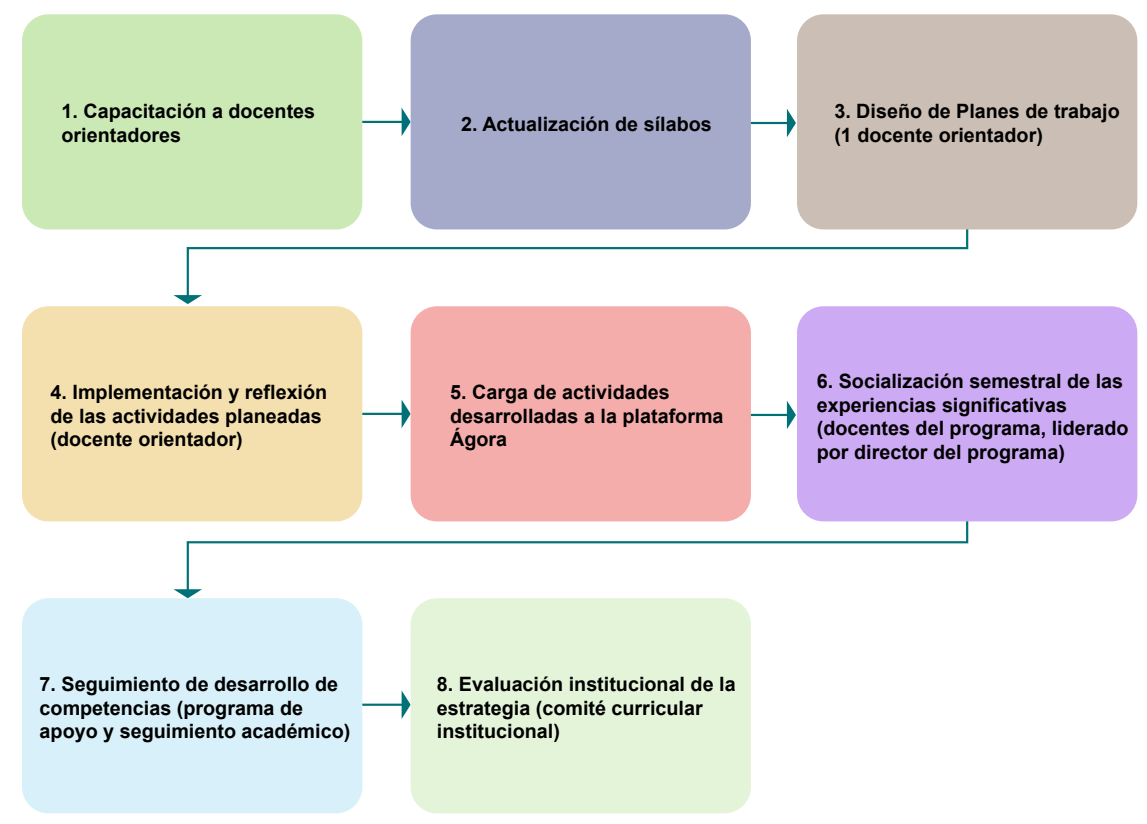

Fuente: elaboración propia.

\section{Conclusiones}

Planear actividades de clase desde las competencias genéricas implica para un docente tener en cuenta el aprendizaje que se espera obtener en los estudiantes, evidenciado mediante desempeños de competencias. En esta investigación, que buscó obtener coherencia entre lo enseñado y lo evaluado, se muestra una posibilidad para ello, que corresponde con asumir las competencias genéricas formuladas por el MEN, agrupadas en conceptuales, procedimentales y actitudinales, y relacionarlas con las competencias genéricas evaluadas por el Icfes, tomando de este último los desempeños y 
evidencias, y adaptándolos en su formulación a las unidades temáticas trabajadas. De esta forma el docente, teniendo presente qué desempeños espera lograr, diseña las actividades de manera que su desarrollo (tanto en clase, como de forma autónoma) pueda dar cuenta de lo aprendido de acuerdo con los criterios de evaluación establecidos en coherencia con los desempeños formulados.

A partir de la generación de la estrategia con los docentes del programa de Licenciatura en Educación Infantil se pudo evidenciar de parte de estos una clara intención de potenciar competencias genéricas a través de contenidos conceptuales planteados, especialmente la lectura crítica y la escritura, seguidos de los contenidos actitudinales, donde se resalta el énfasis en el trabajo en equipo, la participación y liderazgo, y menor atención hacia los contenidos procedimentales, que son aquellos que se enfocan en el cómo ejecutar las habilidades intelectuales y motrices que le permiten al estudiante el aprendizaje. Es así como, mediante los procesos de reflexión por parte de docentes y estudiantes acerca de la experiencia obtenida en la implementación de las actividades planeadas, se observa una tendencia arraigada por parte de los docentes de encauzar su labor hacia el desarrollo de las actividades planeadas sin priorizar la forma de evidenciar el aprendizaje de los estudiantes mediante los desempeños formulados; esta afirmación se fundamenta en que los instrumentos usados no dan cuenta de la manifestación de las competencias diseñadas, sin embargo se espera que la continuidad de este proceso permita evidenciar los resultados por medio de la evaluación externa.

La experiencia de orientación a los docentes del Programa de Licenciatura en Educación Infantil demuestra que para ampliar esta experiencia de forma institucional se requiere la disponibilidad de tiempos y espacios para que los docentes puedan diseñar y reflexionar sobre el desarrollo de desempeños en competencias genéricas, de manera que la planeación de clases a partir de las competencias genéricas sea un proceso al que se le pueda dar la continuidad que les permita a los docentes involucrados el refinamiento del proceso y la autonomía para no depender de un orientador en la construcción de la planeación semestral. 
Una estrategia institucional para el fortalecimiento de las competencias genéricas requiere de la consolidación de un equipo de trabajo que actualice los sílabos y diseñe planes de trabajo acordes al desarrollo de las competencias, así como del compromiso de los docentes en el diseño, implementación y reflexión de la actividad pedagógica coherente con las categorías de análisis halladas en la presente investigación: actividades, criterios de evaluación, instrumento de evaluación y desempeños de las competencias genéricas. Adicionalmente, es fundamental el apoyo del director de programa para la gestión académica y facilitar el aporte que desde del programa de apoyo y seguimiento académico se puede ofrecer para para la realización de simulacros, registro de información y formulación de planes de mejoramiento necesarios en el desarrollo de la estrategia.

\section{Referencias bibliográficas}

Achury, R. (2012). El currículo: reflejo histórico y actual de la educación. Recuperado de https://es.scribd.com/doc/13021882/El-curriculoReflejo-HistOrico-y-Actual-de-La-EducaciOn

Álvarez, M. (2010). Diseñar el currículo universitario: un proceso de suma complejidad. Signo y Pensamiento, 29(56), 68-85.

Caicedo, J. y Calderón, J. (2016). Currículo: en búsqueda de precisiones conceptuales. Revista Educación y Pensamiento, 23, 57-76.

Bautista, N. P. (2011). Proceso de investigación cualitativa: Epistemología, metodología y aplicaciones. Bogotá: Manual Moderno.

Congreso de Colombia. (8 de febrero de 1994). Ley 115 de 1994: por la cual se expide la ley general de educación. Diario Oficial: 41214.

Dewey, J. (1998). Democracia y educación. Madrid: Morata. 
Fundación Universitaria de San Gil (Unisangil). (2004). Lineamientos institucionales sobre competencias genéricas: base para la revisión curricular y para diseños curriculares en todos los programas académicos de Unisangil.

Fundación Universitaria de San Gil (Unisangil). (2018). Proyecto Educativo Institucional. Recuperado de: http://www.unisangil.edu.co/ index.php?option=com_phocadownload \&view=category $\& i d=1$ : instituci onal\&Itemid=901

Gimeno, J. (1994). El currículum: una reflexión sobre la práctica. Madrid: Morata.

Gimeno, J. (2008). Educar por competencias, ¿qué hay de nuevo? Madrid: Morata.

Gómez, O. Y. A. (2019). Uso y apropiación de las TIC en educación. Revista Interamericana de Investigación, Educación y Pedagogía, RIIEP, 12(1), 253-284.

Gómez, O. Y. A., \& Ostos, O.-L. (2018). El constructivismo y el construccionismo. Revista Interamericana de Investigación, Educación y Pedagogía, RIIEP, 11(2), 115-120.

Hernández, B. y Poncio, C. (2018). Educación, desarrollo e innovación social. Bogotá: Andavira .

Icfes. (2020). Valor agregado y aporte relativo. Recuperado de https:// www.icfes.gov.co/web/guest/valor-agregado-y-aporte-relativo

Mertens, L. (2000). La gestión por competencia laboral en la empresa y la formación profesional. Madrid: Organización de Estados Iberoamericanos para la Educación, la Ciencia y la Cultura (OEI).

Ministerio de Educación Nacional. (2006). Lineamientos para la Acreditación de Programas. Recuperado de https://www.mineducacion. gov.co/CNA/1741/articles-186359_lineamientos_2.pdf 
Ministerio de Educación Nacional. (2009). Educación superior. Boletín Informativo, 13. Recuperado de https://www.mineducacion.gov. co/1621/articles-92779_archivo_pdf_Boletin13.pdf

Ostos, O. L. (2017). Visibilidad e impacto de la producción académica. Revista Interamericana de Investigación, Educación y Pedagogía, RIIEP, 10(2), 9-12.

Pansza, M. (1990). Notas sobre planes de estudio y relaciones disciplinares en el currículo. Perfiles Educativos, 36, 16-34.

Pérez, M., Roa, C., Villegas, L. y Vargas, A. (2013). Escribir las prácticas: una propuesta metodológica para planear, analizar, sistematizar y publicar el trabajo didáctico que se realiza en las aulas. Bogotá: Pontificia Universidad Javeriana.

Proyecto Tuning. (2013). Competencias genéricas en América Latina. Recuperado de http://www.tuningal.org/index.php?option=com_conte nt\&view=article\&id=211\& Itemid $=222$

Stenhouse, L. (1984). Investigación y desarrollo del currículo. Madrid: Morata.

Santiváñez, V. (2013). Diseño curricular a partir de competencias. Bogotá: Ediciones de la U.

Schön, D. (1992). La formación de profesionales reflexivos: hacia un nuevo diseño de la enseñanza y el aprendizaje en las profesiones. Barcelona: Paidós.

Tobón, S. (2008). La formación basada en competencias en la educación superior: el enfoque complejo. Documento presentado en el Curso IGLU 2008, Universidad Autónoma de Guadalajara, México.

Zabalza, M. (1987). Diseño y desarrollo curricular. Madrid: Narcea.

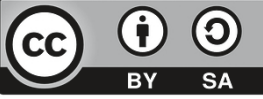

